\title{
Equisizable partial sum families
}

\author{
Ricardo Grande ${ }^{1}$ István Kovács ${ }^{2}$ - Klavdija Kutnar ${ }^{2}$ - Aleksander Malnič ${ }^{\text {. }}$ \\ Luis Martínez ${ }^{4,5}$. Dragan Marušič ${ }^{2,3}$
}

Received: 14 February 2017 / Accepted: 28 January 2019 / Published online: 26 March 2019

(c) Springer Science+Business Media, LLC, part of Springer Nature 2019

\begin{abstract}
We introduce a special kind of partial sum families, which we call equisizable partial sum families, that can be used to obtain directed strongly regular graphs admitting a semiregular group of automorphisms. We give a construction of an infinite family of equisizable partial sum families depending on two parameters that produce directed strongly regular graphs with new parameters. We also determine the automorphisms group of the associated directed strongly regular graphs in terms of the parameters.
\end{abstract}

Keywords Directed strongly regular graphs · Groups of automorphisms · Partial sum families

\section{Introduction}

Strongly regular graphs were introduced by Bose [3] in 1963 as regular graphs in which the number of common neighbours of two vertices depends only on whether the vertices are adjacent or not. This concept was later generalized to directed graphs by Duval [5] in 1988. He defined a directed strongly regular graph with parameters $(v, k, \mu, \lambda, t)$ [or just $(v, k, \mu, \lambda, t)$-DSRG, for short], as a directed graph of order $v$ in which all vertices have in-valency and out-valency $k$, and for every two vertices $u$ and $w$, the number of paths of length 2 beginning in $u$ and ending in $w$ is $t, \lambda$ or $\mu$ according to whether $u=w, u w$ is an arc or $u \neq w$ and $u w$ is not an arc.

\footnotetext{
Luis Martínez was supported by the Basque Government Grant IT974-16 and by the UPV/EHU and Basque Center of Applied Mathematics, grant US18/21. The work of István Kovács was supported in part by the Slovenian Research Agency (Research Program P1-0285 and Research Projects N1-0032, N1-0038, N1-0062, J1-6720, J1-7051, J1-9108). The work of Klavdija Kutnar was supported in part by the Slovenian Research Agency (Research Program P1-0285 and Research Projects N1-0032, N1-0038, J1-6720, J1-6743, J1-7051, J1-9110), in part by WoodWisdom-Net+, $\mathrm{W}^{3} \mathrm{~B}$, and in part by NSFC Project 11561021. The work of Dragan Marušič was supported in part by the Slovenian Research Agency (I0-0035, Research Program P1-0285 and Research Projects N1-0032, N1-0038, N1-0062, J1-5433, J1-6720, J1-7051, J1-9108) and in part by H2020 Teaming InnoRenew CoE (Grant No. 739574).
}

Extended author information available on the last page of the article 
Martínez and Araluze studied in [11] the necessary and sufficient conditions for the existence of a $(v, k, \mu, \lambda, t)$-DSRG admitting a semiregular group $H$ of automorphisms. They proved that its existence is equivalent to the existence of an algebraic combinatorial structure that they called partial sum family (or PSF, for short), formed by an array of subsets (also called blocks) of an extension $G$ of $H$. The symbol of the associated directed strongly regular graph can be deduced from the blocks of the partial sum family, and the size of the array of blocks is the number of orbits of the action. Partial sum families were later studied in more detail in $[1,2,12]$.

In this paper, we will present a special kind of partial sum families, which we call equisizable partial sum families, characterized by the property that the size of the blocks in each column is the same, except for the block in the last row of the column. In Sect. 2, we introduce equisizable PSFs and we establish some of their basic properties. In Sect. 3, we obtain one of the two main results of the paper: the construction of an infinite family of equisizable partial sum families depending on two parameters. The existence of the associated family of directed strongly regular graphs was previously undecided in many cases. Finally, in Sect. 4 we obtain our second main result: the description of the full automorphism groups of the infinite family of directed strongly regular graphs that are obtained in Sect. 3 .

\section{Terminology and first properties}

Given integers $m \geq 1$ and $n \geq 2$, a group of automorphisms of a digraph is called $(m, n)$-semiregular if it has $m$ orbits of length $n$ and no other orbit, and the action is regular on each orbit. An $m$-Cayley digraph $\Gamma$ is a digraph admitting an $(m, n)$-semiregular group $H$ of automorphisms. Every $m$-Cayley digraph $\Gamma$ can be represented, following the terminology established in [10], by an $m \times m$ array of subsets of $H$ in the following way. Let $U_{0}, \ldots, U_{m-1}$ be the $m$ orbits of $H$, and for each $i$ let $u_{i} \in U_{i}$. For each $i$ and $j$, let $S_{i, j}$ be defined by $S_{i, j}=\left\{h \in H \mid u_{i} u_{j}^{h} \in A(\Gamma)\right\}$, where $A(\Gamma)$ denotes the arc set of $\Gamma$, and we write $u v$ for the arc (or directed edge) pointing from $u$ to $v$. The family $\left(S_{i, j}\right)$ is called the symbol of $\Gamma$ relative to $\left(H ; u_{0}, \ldots, u_{m-1}\right)$.

A directed graph $\Gamma$ without loops, of valency $k$ and order $v$, as defined by Duval [5], is called a directed strongly regular graph with parameters $(v, k, \mu, \lambda, t)$ (for short, $(v, k, \mu, \lambda, t)$-DSRG or simply DSRG if we do not specify the parameters) whenever for any vertex $u$ of $\Gamma$ there are $t$ undirected edges having $u$ as an end vertex and for every two different vertices $u$ and $w$ of $\Gamma$ the number of paths of length 2 starting at $u$ and ending at $w$ equals $\lambda$ if $u w \in A(\Gamma)$ and equals $\mu$ if $u w \notin A(\Gamma)$.

A directed strongly regular graph will also be referred to as a strongly regular digraph.

The following relation between the parameters of a DSRG can be found in [5]:

$$
k(k-\beta)=\mu v+\gamma,
$$

where $\beta=\lambda-\mu$ and $\gamma=t-\mu$. 
It is proved in [5] that $\beta^{2}+4 \gamma$ is a square, unless

$$
k=t=(v-1) / 2, \quad \mu=(v-1) / 4, \quad \lambda=(v-5) / 4,
$$

in which case the graph is undirected and is a conference graph, or

$$
k=(v-1) / 2, \quad \mu=(v+1) / 4, \quad \lambda=(v-3) / 4, \quad t=0 .
$$

We define

$$
\Delta=\sqrt{\beta^{2}+4 \gamma}
$$

which is an integer if the parameters of the digraph are not as indicated above. We will assume in the rest of the paper that this is the case.

The following definition of partial sum family was given in [11] in a general setting, and in a specialized form later in [1] in the way that we present here for abelian groups. Condition (iii) of the definition represents an identity in the group ring $\mathbb{Z}[H]$ where, as usual, a subset of $H$ is identified with the sum in $\mathbb{Z}[H]$ of its elements (for definitions and results on group rings, we refer the reader to [13]). As the considered group $H$ is abelian and additive notation is often used in abelian groups, we write $H$ additively and denote its operation by + . In order to avoid confusion, the addition in the group ring $\mathbb{Z}[H]$ will be denoted by $\oplus$, and regarding the fact that the multiplication in $\mathbb{Z}[H]$ extends the group operation of $H$, this will also be denoted by + .

Definition 2.1 Let $H$ be an abelian group of order $n$ and let $m$ be an integer with $m \geq 1$. A family $\mathfrak{S}=\left(S_{i, j}\right)$, with $0 \leq i, j<m$, of subsets of $H$ is a $(m, n, k, \mu, \lambda, t)$-partial sum family (for short, $(m, n, k, \mu, \lambda, t)$-PSF, or simply PSF if we do not specify the parameters) if it satisfies:

(i) for every $i$ it holds that $e \notin S_{i, i}$, where $e$ is the identity of $H$.

(ii) for every $i$ it holds that $\sum_{j=0}^{m-1}\left|S_{i, j}\right|=\sum_{j=0}^{m-1}\left|S_{j, i}\right|=k$.

(iii) for every $i$ and $j$ the following identity holds in the group ring $\mathbb{Z}[H]$ :

$$
\sum_{l=0}^{m-1} \oplus\left(S_{i, l}+S_{l, j}\right)=\delta_{i, j} \gamma e \oplus \beta S_{i, j} \oplus \mu H,
$$

where $\delta_{i, j}$ is the Kronecker delta, and where $\gamma=t-\mu$ and $\beta=\lambda-\mu$.

In the rest of the paper, we will write $\Delta=\sqrt{\beta^{2}+4 \gamma}$ when working with PSFs, following the notation used for DSRGs.

In the following obvious result, which is stated with more generality for arbitrary groups in Proposition 1.2 in [1], the connection of partial sum families with directed strongly regular graphs admitting abelian semiregular groups of automorphisms is presented: 
Proposition 2.2 For an abelian group $H$ of order $n$, a family $\mathfrak{S}=\left(S_{i, j}\right), 0 \leq i, j<$ $m$, of subsets of $H$ is an $(m, n, k, \mu, \lambda, t)$-PSF iff the digraph associated with the symbol $\left(S_{i, j}\right)$ is an $(m n, k, \mu, \lambda, t)$-DSRG, which admits $H$ as an $(m, n)$-semiregular group of automorphisms.

Remark 2.3 The associated digraph mentioned in the previous proposition has vertex set

$$
V=\left\{h_{i} \mid h \in H, 0 \leq i \leq m-1\right\}
$$

constituted by elements of the group $H$ indexed by elements of the set $\{0, \ldots, m-1\}$ and has arc set

$$
A=\left\{h_{i} h_{j}^{\prime} \mid 0 \leq i, j \leq m-1, h^{\prime}-h \in S_{i, j}\right\} .
$$

Now the following identity for the parameters of any PSF follows immediately from (1):

$$
k(k-\beta)=\mu n m+\gamma .
$$

We will denote the cardinality of $S_{i, j}$ by $s_{i, j}$.

Proposition 2.4 If $\mathfrak{S}$ is a PSF on an abelian group $H$ and $\chi$ is a non-trivial character of $H$, then

$$
\sum_{j=0}^{m-1} \chi\left(S_{j, j}\right)=m(\beta-\Delta) / 2+i \Delta
$$

for some $i=0, \ldots, m$.

Proof By applying the character $\chi$ in part (iii) of Definition 2.1, we conclude that the matrix

$$
A_{\chi}=\left(\chi\left(S_{i, j}\right)\right)
$$

satisfies $A_{\chi}^{2}=\beta A_{\chi}+\gamma I_{m}$, and hence, its trace $\sum_{j=0}^{m-1} \chi\left(S_{j, j}\right)$ is the sum of $m$ roots of $x^{2}-\beta x-\gamma$. Since the roots of this polynomial are $\frac{1}{2}(\beta \pm \Delta)$, the result follows immediately.

The following is our main definition, in which we introduce the kind of PSFs that we will study later.

Definition 2.5 A PSF with $m \geq 3$ is equisizable if $s_{i, j}=s_{0, j}$ for all $i \leq m-2$ and for all $j \leq m-1$.

Observe that then

$$
s_{m-1, j}=k-(m-1) s_{0, j} .
$$

In an equisizable PSF, we will denote the common value of the $s_{i, j}$ with $i \leq m-2$ simply by $s_{j}$. 
Proposition 2.6 If $\mathfrak{S}$ is an equisizable PSF, then $\gamma=0$.

Proof By taking the trivial character in part (iii) of Definition 2.1, we have

$$
\sum_{l=0}^{m-1} s_{i, l} s_{l, j}=\delta_{i, j} \gamma+\beta s_{i, j}+\mu n
$$

If $i<m-1$, since the PSF is equisizable we have

$$
\sum_{l=0}^{m-2} s_{l} s_{j}+s_{m-1}\left(k-(m-1) s_{j}\right)=\gamma \delta_{i, j}+\beta s_{j}+\mu n
$$

and, factoring out $s_{j}$ we obtain

$$
k\left(s_{j}+s_{m-1}\right)-m s_{m-1} s_{j}=\gamma \delta_{i, j}+\beta s_{j}+\mu n .
$$

Since the left-hand side of the previous identity does not depend on $i$, by taking $i=0, j=0$ and $i=1, j=0$ and subtracting we obtain the desired result.

Proposition 2.7 If $\mathfrak{S}$ is an equisizable PSF, then one of the following two possibilities holds:

(i) $s_{j}=k / m$ for every $j$,

(ii) $s_{m-1}=\mu n / k$.

Proof Taking $j=m-1$ in (6) and using that $\gamma=0$, we obtain

$$
m s_{m-1}^{2}+(\beta-2 k) s_{m-1}+\mu n=0 .
$$

Solving the equation for $s_{m-1}$, we get

$$
s_{m-1}=\left(2 k-\beta \pm \sqrt{(\beta-2 k)^{2}-4 \mu n m}\right) /(2 m),
$$

and using (4), we obtain

$$
s_{m-1}=(2 k-\beta \pm \beta) /(2 m) \text {. }
$$

If the minus sign holds, we obtain $s_{m-1}=\mu n / k$, as stated in part (ii) of the proposition. Let us suppose that the plus sign holds. Substituting $s_{m-1}$ in (6), we have $s_{j}=$ $\left(k^{2}-\mu n m\right) /(m \beta)$, and using (4) we deduce that $s_{j}=k / m$ for all $j$, as stated in part (i) of the proposition.

We will say that a PSF is of type 1 if (i) holds in the previous proposition, and that is of type 2 otherwise.

The following theorem, which is given in Proposition 3.5 in [2] without using the terminology of equisizable PSFs, guarantees that equisizable PSFs of type 1 always exist: 
Theorem 2.8 For every $n, m$ in $\mathbb{N}$ such that $m$ divides $n-1$, there exists a DSRG with parameters

$$
v=n m, \quad k=n-1, \quad \mu=t=(n-1) / m, \quad \lambda=(n-1) / m-1
$$

admitting a semiregular group of automorphisms with $m$ orbits associated with an equisizable PSF of type 1.

Now we will focus our attention in the PSFs of type 2.

Lemma 2.9 Let $H$ be a cyclic group of order $n$, and let $A=\sum_{h \in H}^{\oplus} a_{h} h$ be an element of the group ring $\mathbb{Z}[H]$. If there exists a set I of $r$ integers in arithmetic progression with difference $d$ such that $\chi(A) \in I$ for every non-trivial character $\chi$ of $H$, then the following hold:

(i) There exist integers $c_{n^{\prime}}$ such that $A=\sum_{n^{\prime} \mid n}^{\oplus} c_{n^{\prime}} U_{n^{\prime}}$, where $U_{n^{\prime}}$ denotes the unique subgroup of order $n^{\prime}$ of $H$.

(ii) If $n^{\prime} \neq 1, n$ then $c_{n^{\prime}}$ has the form $c_{n^{\prime}}=\frac{d w_{n^{\prime}}}{n^{\prime}}$ with $w_{n^{\prime}} \in \mathbb{Z}$, and $w_{n^{\prime}} \neq 0$ only if $n^{\prime}$ divides $u d$, where $u=1 . c . m .\{2,3, \ldots, r-1\}$.

Proof Both statements can be derived along the line of the proof of a part of Lemma 3.5 in [4] (in fact, that part is the case when $r=3$ ), and hence, the proof is omitted.

When $r=4$, the lemma reduces to Lemma 5.1 in [9]; thus, our lemma is a generalization of the two mentioned ones.

Proposition 2.10 Let $H$ be a cyclic group, and let $\mathfrak{S}$ be $a(m, n, k, \mu, \lambda, t)$-PSF over the group $H$. If one of the two following conditions is satisfied:

(i) $n$ is a prime,

(ii) $n$ is coprime to $m ! \Delta$,

then $\left(S_{j, j}\right)_{j \leq m-1}$ covers all the elements of $H-\{e\}$ the same number of times.

Proof By using Proposition 2.4, we can see that the hypotheses of the previous lemma are satisfied for $A=\sum^{\oplus} S_{j, j}, r=m+1$ and $d=\Delta$. Now, if $n$ is a prime, the result follows easily from the first part of the lemma, and if it is coprime to $m ! \Delta$, then it follows from the second part.

The proposition that we have just proved shows that very often the 'diagonal blocks' of a PSF cover the non-trivial elements of the group the same number of times. Next we will see that, when this is the case, we can determine the parameters $(m, n, k, \mu, \lambda, t)$ for the equisizable PSFs of type 2 .

Proposition 2.11 If $\mathfrak{S}$ is an equisizable $(m, n, k, \mu, \lambda, t)$-PSF of type 2 over a cyclic group satisfying $\sum_{j=0}^{m-1} \oplus S_{j, j}=\delta(H \ominus\{e\})$ (with the obvious interpretation for the symbol $\ominus$ ) in the group ring $\mathbb{Z}[H]$ for some $\delta \in \mathbb{N}$, then:

(i) $k=\delta n, \quad \mu=t=\delta^{2}(n+1) / m, \quad \lambda=\delta^{2}(n+1) / m-\delta$, or 
(ii) $k=\delta n-\delta / 2, \mu=t=\delta(\delta n-\delta / 2) / m, \lambda=\delta(\delta n-\delta / 2) / m-\delta / 2($ only if $\delta$ is even), or

(iii) $(m, n, k, \mu, \lambda, t) \in \mathfrak{P}_{\delta}$, where $\mathfrak{P}_{\delta}$ is a finite set which depends only on $\delta$.

Proof Taking the trivial character in $\sum_{j=0}^{m-1} \oplus S_{j, j}=\delta(H \ominus\{e\})$, and using (5), the fact that the PSF is of type 2 and (4), we obtain

$$
\delta(n-1)=2 k-\mu n m / k=k+\beta .
$$

By taking now a non-trivial character and using Proposition 2.4, we obtain $-\delta=$ $m(\beta-\Delta) / 2+i^{\prime} \Delta$ for some $i^{\prime}=0, \ldots, m$. We have $\Delta=|\beta|$, and since $-\delta<0$, we must have $\Delta=-\beta$, and $-\delta=\left(m-i^{\prime}\right) \beta$ holds. Putting $i=m-i^{\prime}$, and having into account that $\delta \neq 0$, we conclude that $-\delta=i \beta$ for some $i=1 \ldots, m$. If $\beta=-\delta$, it follows from (7) that the parameters have the form stated in the first case of the proposition.

Let us assume that $\beta \neq-\delta$. Using (7), we get $n=\frac{k+\beta+\delta}{\delta}$. From (4), it follows that:

$$
m \mu=\frac{\delta k(k-\beta)}{k+\beta+\delta} .
$$

If $k-\beta=k+\beta+\delta$, then $\delta=-2 \beta$. Using (7), we get the parameters stated in the second case of the proposition.

Therefore, we will suppose that $k-\beta \neq k+\beta+\delta$, and thus $-\delta=i \beta$ for $i>2$. It follows that $\beta<0$, and it is easy to deduce that $\delta>\beta+\delta>2 \beta+\delta>0$.

Now we will prove that no prime $p>\delta$ divides $k+\beta+\delta$. Let us suppose that such a prime $p$ exists. Then $p$ divides $\delta k(k-\beta)$. Of course, $p$ does not divide $\delta$. It does not divide $k-\beta$ either, as it is not difficult to see that $k+\beta+\delta$ and $k-\beta$ have different residues modulo $p$. Therefore, $p$ must divide $k$. But this gives a contradiction, because $k+\beta+\delta$ and $k$ have different residues modulo $p$.

Therefore, there is no prime $p>\delta$ that divides $k+\beta+\delta$. Thus, $k+\beta+\delta=\prod_{j} p_{j}^{\alpha_{j}}$ where $p_{j}$ are primes such that $2 \leq p_{j} \leq \delta$. Using that $k+\beta+\delta$ divides $\delta k(k-\beta)$, it is easy to deduce that:

$$
\alpha_{j} \leq v_{p_{j}}(\delta)+v_{p_{j}}(\delta+\beta)+v_{p_{j}}(\delta+2 \beta)
$$

where $v_{p}(z)$ denotes the $p$-adic valuation of an integer $z$ (that is, the largest exponent $e$ that $p^{e}$ divides $z$ if $z \neq 0$, and $\infty$ for $z=0$ ).

Thus, there is a finite number of possible values for $k+\beta+\delta$. It follows that there is a finite number of possible values for $k$ as well. As $m$ must divide $\frac{\delta k(k-\beta)}{k+\beta+\delta}$, there is a finite number of possible values of $m$ for each $k$. To sum up, $(m, n, k, \mu, \lambda, t) \in \mathfrak{P}_{\delta}$, where $\mathfrak{P}_{\delta}$ is a finite set.

It is important to point out that given $\delta$, the family $\mathfrak{P}_{\delta}$ can be completely determined by using the divisibility conditions explained in the proof. When $\delta=1$, we only get the first infinite family of the proposition. When $\delta=2$, we get the two infinite families 
of the proposition. When $\delta=3$, the second family is not possible, so we would get the first one and $\mathfrak{P}_{3}$. We finish the section by determining the parameters in $\mathfrak{P}_{3}$.

First of all, we have two equations:

$$
3(n-1)=k+\beta, \quad-3=i \beta \quad i \in\{1, \ldots, m\} .
$$

Thus, $i=1, \beta=-3$ or $i=3, \beta=-1$. The first solution corresponds to the first infinite family, so we turn to the second one. Following the ideas of the proof, we obtain

$$
n=\frac{k+2}{3}, \quad m \mu=\frac{3 k(k+1)}{k+2}
$$

As $k+1$ and $k+2$ are coprime, we have that $k+2$ divides $3 k$. If $p$ is a prime that divides $k+2$, it must satisfy $2 \leq p \leq \delta=3$ so $p=2$, 3. Thus, $k+2=2^{s} 3^{s^{\prime}}$. Also, $k+2$ divides $3 k$ so $s, s^{\prime} \leq 1$. We get $k \leq 4$ and taking into account that $k+2=2^{s} 3^{s^{\prime}}$, $k=1$, 4. Using (8), we get:

$$
\begin{array}{ll}
k=1, & n=1, \quad \mu=t=2 / m, \quad \lambda=2 / m-1 \\
k=4, \quad n=2, \quad \mu=t=10 / m, \quad \lambda=10 / m-1
\end{array}
$$

However, we are going to prove below that none of these parameters gives DSRGs satisfying

$$
\sum_{i=0}^{m-1} \oplus S_{i, i}=3(H \ominus\{e\}) .
$$

In particular, the first parameters do not give DSRGs as the smallest DSRG is of order 6 . The same argument shows that for the second set of parameters we must have $m \in\{5,10\}$. Let $\Gamma$ be the DSRG defined by the symbol $\left(S_{i, j}\right)$ having these parameters, and let the orbits of $H$ on the vertex set $V(\Gamma)$ of $\Gamma$ be denoted by $U_{i}$, $i \in \mathbb{Z}_{m}$. Using that $\delta=3$ and $n=2$, we may assume without of loss of generality that $S_{0,0}=S_{1,1}=S_{2,2}=H-\{e\}=\{1\}$, and $S_{i, i}=\emptyset$ for all $i>2$.

Suppose first that $m=5$. Then the parameters are $k=4, n=2, \mu=t=2, \lambda=$ 1 , and $v=n m=10$. Since $t=2$, the undirected edges form a spanning subgraph of $\Gamma$ isomorphic to a union of cycles. If the orbit $U_{3}$ is not adjacent to $U_{4}$ with an undirected edge, then it must be adjacent with undirected edges to two of the orbits $U_{0}, U_{1}$ and $U_{2}$; without loss of generality, we may assume that these two orbits are $U_{0}$ and $U_{1}$. Applying the action of $H$, one can see that the undirected subgraph of $\Gamma$ induced on $U_{0} \cup U_{1} \cup U_{3}$ is a 6-cycle, and consequently, the orbits $U_{2}$ and $U_{4}$ must induce a 4-cycle, which is clearly impossible as $S_{4,4}=\emptyset$. If, however, the orbit $U_{3}$ is adjacent to $U_{4}$ with an undirected edge, then the undirected subgraph of $\Gamma$ induced on $U_{3} \cup U_{4}$ is either a 4-cycle or $2 K_{2}$. However, applying the action of $H$ one can see that none of these two cases produces a spanning undirected subgraph of $\Gamma$ isomorphic to a union of cycles.

Suppose now that $m=10$. Since $t=1$, the undirected edges of $\Gamma$ must induce a perfect matching of $\Gamma$. Three undirected edges lie inside the orbits $U_{i}, i=0,1,2$, 
and the other edges have end vertices in different orbits of $H$. Applying the action of $H$, one can see that between two orbits of $H$ that are adjacent with an undirected edge we must have $2 K_{2}$, implying that there is a 1 -factor spanned by seven orbits of $H$, a contradiction.

The final part of our previous analysis can be summarized in the following theorem:

Theorem 2.12 There do not exist equisizable DSRGs with parameters $(10,4,2,1,2)$ and $(20,4,1,0,1)$ admitting semiregular groups of automorphisms with 5 and 10 orbits, respectively, associated with equisizable PSFs of type 2.

The theorem shows that, in the first case in which $\mathfrak{P}_{\delta}$ is not empty, that is, when $\delta=3$, there exists no PSF with the corresponding feasible parameters of tuples in $\mathfrak{P}_{3}$. For greater values of $\delta$, the complexity of $\mathfrak{P}_{\delta}$ augments, and it would be an interesting question to know whether there exist PSFs for those parameters.

\section{A construction of equisizable PSFs}

When $\delta=1$, Proposition 2.11 guarantees that the parameters of our equisizable PSF of type 2 over a cyclic group $H$ must be

$$
k=n, \quad \mu=t=(n+1) / m, \quad \lambda=(n+1) / m-1,
$$

and we can re-parametrize it in the following way (by calling $\mu=t=\alpha$ ):

$$
n=k=\alpha m-1, \quad \mu=t=\alpha, \quad \lambda=\alpha-1 .
$$

Although we know the parameters, we still do not know if such a PSF exists. This section is devoted to the construction of such an equisizable PSF whose parameters are the ones mentioned above. First, let us denote the elements of the cyclic group $H=\mathbb{Z}_{\alpha m-1}$ as follows:

$$
H=\{0,1,2, \ldots, \alpha m-3, \alpha m-2\},
$$

and the group operation of $H$ is the addition modulo $n=\alpha m-1$. Let us set

$$
\begin{aligned}
& y_{0}=y_{1}=z_{0}=z_{1}=0 \\
& y_{l}=\sum_{i=1}^{l-1} i, \quad z_{l}=-y_{l}, \quad l=2, \ldots, m-2 .
\end{aligned}
$$

Moreover, let us define the following subsets in $H$ :

$$
\begin{aligned}
& A=\{s(m-1) \mid s=1, \ldots, \alpha-1\} \\
& B=\{s m-(s-1) \mid s=0, \ldots, \alpha-1\} \\
& C=\left(-\alpha-z_{m-2}\right)+\{0,1, \ldots, m+\alpha-3\} \\
& D=\left(-z_{m-2}\right)+\{\alpha m-\alpha+s \mid s=0, \ldots, \alpha-2\}
\end{aligned}
$$


where if $\lambda \in H$ and $E \subset H$, then $\lambda+E=\{\lambda+e \mid e \in E\}$.

This definition is valid for any positive integer $\alpha$, even when $\alpha=1$ if we understand that $A=D=\varnothing$ in that case.

Finally, let us consider the following family of subsets of $H$, arranged in an $m \times m$ matrix:

$$
\left(\begin{array}{c|cccc}
y_{0}+A & y_{0}+z_{0}+B & y_{0}+z_{1}+B & \ldots & y_{0}+z_{m-2}+B \\
y_{1}+A & y_{1}+z_{0}+B & y_{1}+z_{1}+B & \ldots & y_{1}+z_{m-2}+B \\
\vdots & \vdots & \vdots & \ddots & \vdots \\
y_{m-2}+A & y_{m-2}+z_{0}+B & y_{m-2}+z_{1}+B & \ldots & y_{m-2}+z_{m-2}+B \\
\hline C & z_{0}+D & z_{1}+D & \ldots & z_{m-2}+D
\end{array}\right)
$$

In order to check that this is actually a PSF, we need to go through the three conditions in Definition 2.1. The first and second conditions are easy to check just from the definition of $A, B, C$ and $D$. The next proposition makes it easier to check the third condition. We omit the proof, which is standard and only requires the definition of the sets $A, B, C$ and $D$ and the elements $y_{i}$ and $z_{j}$.

Proposition 3.1 The $m^{2}$ equations listed in the third condition of Definition 2.1 are equivalent to the following four equations for our family:

(i) $(A+A) \oplus \sum_{l=0}^{m-3} \oplus\left(y_{l+1}+z_{l}+A+B\right) \oplus\left(z_{m-2}+B+C\right)=\beta A \oplus \mu H$

(ii) $(A+B) \oplus \sum_{l=0}^{m-3} \oplus\left(y_{l+1}+z_{l}+B+B\right) \oplus\left(z_{m-2}+B+D\right)=\beta B \oplus \mu H$

(iii) $(A+C) \oplus \sum_{l=0}^{m-3} \oplus\left(y_{l+1}+z_{l}+A+D\right) \oplus\left(z_{m-2}+C+D\right)=\beta C \oplus \mu H$

(iv) $(B+C) \oplus \sum_{l=0}^{m-3} \oplus\left(y_{l+1}+z_{l}+B+D\right) \oplus\left(z_{m-2}+D+D\right)=\beta D \oplus \mu H$

The following result proves that what we have defined is a PSF. We will only give a sketch of the proof as it is only based on appropriate use of the definition of the sets $A, B, C$ and $D$ and the elements $y_{i}$ and $z_{j}$.

Proposition 3.2 The sets A, B, C and D satisfy the equations given in Proposition 3.1.

Proof The following identities are easy to check and come in handy during the proof.

$$
\begin{aligned}
& (\{0,1, \ldots, m-3\}+B) \oplus A=H \ominus\left(z_{m-2}+D\right) \ominus\{0\} \\
& B=(1+A) \oplus\{1\}
\end{aligned}
$$

We will prove that the equation in case (i) of Proposition 3.1 is satisfied. We begin by writing the equation, and we will manipulate it until we find an expression which we know is true.

$$
\begin{aligned}
& (A+A) \oplus \sum_{l=0}^{m-3} \oplus\left(y_{l+1}+z_{l}+A+B\right) \oplus\left(z_{m-2}+B+C\right)=\beta A \oplus \mu H \\
& (A+A) \oplus(\{0,1, \ldots, m-3\}+A+B) \oplus\left(z_{m-2}+B+C\right)=\ominus A \oplus \alpha H
\end{aligned}
$$


Now we use identity (9),

$$
\begin{aligned}
& A+\left(A \oplus H \ominus A \ominus\left(z_{m-2}+D\right) \ominus\{0\}\right) \oplus\left(z_{m-2}+B+C\right)=\ominus A \oplus \alpha H \\
& (A+H) \ominus\left(z_{m-2}+D+A\right) \oplus\left(z_{m-2}+B+C\right)=\alpha H
\end{aligned}
$$

By re-arranging the terms and using the definition of $C$ and $D$, we find:

$$
(-\alpha)+\{0, \ldots, m+\alpha-3\}+B=(\{\alpha m-\alpha, \ldots, \alpha m-2\}+A) \oplus H
$$

and we deduce that

$$
\{0, \ldots, m+\alpha-3\}+B=(\{1, \ldots, \alpha-1\}+A) \oplus H
$$

Now we use identity (10),

$$
\begin{aligned}
& \{0, \ldots, m+\alpha-3\}+B=\{1, \ldots, \alpha-1\}+([(-1)+B] \ominus\{0\}) \oplus H \\
& ((\alpha-1)+\{0, \ldots, m-2\}+B) \oplus\{1, \ldots, \alpha-1\}=H \\
& ((\alpha-1)+(H \ominus\{\alpha m-\alpha+1, \ldots, \alpha m-2,0\})) \oplus\{1, \ldots, \alpha-1\}=H \\
& \ominus((\alpha-1)+\{\alpha m-\alpha+1, \alpha m-\alpha+2, \ldots, \alpha m-2,0\}) \oplus\{1, \ldots, \alpha-1\}=\emptyset \\
& \ominus\{1, \ldots, \alpha-1\} \oplus\{1, \ldots, \alpha-1\}=\emptyset
\end{aligned}
$$

which is obviously true, and thus, $A, B, C$ and $D$ satisfy the first equation. The rest are very similar and can be proved using exactly the same procedure.

We will denote by $S(H, \alpha, m)$ the PSF described above, and by $\Gamma(H, \alpha, m)$ the corresponding directed strongly regular graph.

Then, we have proved the following existence result:

Theorem 3.3 For every $n, m$ in $\mathbb{N}$ such that $m$ divides $n+1$, there exists a DSRG with parameters

$$
v=n m, \quad k=n, \quad \mu=t=(n+1) / m, \quad \lambda=(n+1) / m-1
$$

admitting a semiregular group of automorphisms with $m$ orbits associated with an equisizable PSF of type 2.

The construction presented in this section originates DSRGs whose existence, in many cases, was previously unknown. In Table 1, the parameters of 12 DSRGs that appear as undecided in Hobart and Brouwer's table [7] are presented. In fact, considering also the corresponding complements, 24 undecided cases of the mentioned table are proven to exist. Also, the following six sets of parameters of DSRGs, that can be obtained with the mentioned construction, correspond with parameters of DSRGs constructed by Gyürki [6] using a different method:

$(60,15,4,3,4),(60,20,7,6,7),(78,26,9,8,9),(96,32,11,10,11)$, $(105,35,12,11,12),(108,27,7,6,7)$ 
Table 1 Parameters of new directed strongly regular graphs

\begin{tabular}{lll}
\hline$(44,11,3,2,3)$ & $(45,9,2,1,2)$ & $(51,17,6,5,6)$ \\
$(66,11,2,1,2)$ & $(69,23,8,7,8)$ & $(70,14,3,2,3)$ \\
$(76,19,5,4,5)$ & $(87,29,10,9,10)$ & $(91,13,2,1,2)$ \\
$(92,23,6,5,6)$ & $(95,19,4,3,4)$ & $(102,17,3,2,3)$ \\
\hline
\end{tabular}

\section{Automorphism groups}

In this section, we will investigate some properties of the digraphs that we constructed in Sect. 3. In particular, we will derive their automorphism groups in three theorems.

Let $\mathcal{J}(m, 2)$ be a digraph with vertex set

$$
V(\mathcal{J}(m, 2))=\{(i, j) \mid i \in\{0, \ldots, m-1\}, j \in\{0, \ldots, m-1\}-\{i\}\},
$$

in which there is an arc from the vertex $(i, j)$ to the vertex $(k, \ell)$ if and only if $j=k$. Observe that each vertex in this digraph belongs to exactly one undirected edge and that $S_{m} \leq \operatorname{Aut}(\mathcal{J}(m, 2))$ since any permutation of an $m$-set maps disjoint 2-subsets to disjoint 2-subsets. Moreover, $S_{m} \leq \operatorname{Aut}(\mathcal{J}(m, 2))$ acts vertex-transitively, and the set $\mathcal{B}=\{\{(i, j),(j, i)\}: i, j \in\{0, \ldots, m-1\}\}$ gives an invariant partition of the vertex set of $\mathcal{J}(m, 2)$ for the full automorphism group. (Namely, all undirected edges of the digraph connect the two vertices from the same set of this partition, where undirected edges are pairs of parallel oppositely oriented arcs.) Furthermore, one can easily see that the quotient digraph with respect to the partition $\mathcal{B}$ in which we forget about the loops (arising from the undirected edges in $\mathcal{J}(m, 2)$ ) and replace pairs of two parallel oppositely oriented arcs with edges gives the Johnson graph $J(m, 2)$. It is proved in [14] that, if $m \neq 2 k$, then the automorphism group of $J(m, k)$ is isomorphic to the symmetric group $S_{m}$. Using this fact and that $S_{m} \leq \operatorname{Aut}(\mathcal{J}(m, 2))$, we conclude that, if $m \neq 4$, then $S_{m} \cong \operatorname{Aut}(J(m, 2))=\operatorname{Aut}(\mathcal{J}(m, 2))$. It is checked with a direct calculation that $S_{4} \cong \operatorname{Aut}(\mathcal{J}(4,2))$.

Because of this nice relation between the defined family of digraphs and the Johnson graphs, we will call the digraphs $\mathcal{J}(m, 2)$ Johnson digraphs.

The following theorem shows that a DSRG arising from the equisizable PSF we constructed in Sect. 3, with parameters $n=k=m-1, \mu=t=1$ and $\lambda=0$ is isomorphic to $\mathcal{J}(m, 2)$, implying that Johnson digraphs are in fact DSRGs.

Theorem 4.1 The directed graph $\Gamma\left(\mathbb{Z}_{m-1}, 1, m\right)$ is vertex-transitive, and it is isomorphic to $\mathcal{J}(m, 2)$, and thus, $\operatorname{Aut}\left(\Gamma\left(\mathbb{Z}_{m-1}, 1, m\right)\right) \cong S_{m}$.

Proof Remember that the symbol of $\Gamma$ is given by the sets

$$
A=D=\emptyset, \quad B=\{1\} \text { and } C=H
$$

where $H=\mathbb{Z}_{m-1}=\{0, \ldots, m-2\}$, the cyclic group with $m-1$ elements. Let us consider the permutation $\rho=(m-1)(0, \ldots, m-2) \in \operatorname{Sym}(\{0,1, \ldots, m-1\})$. Let $X=\mathcal{J}(m, 2)$. Clearly, $\rho$ is an automorphism of the graph $X$ acting in the obvious way on the vertex set of $X:(i, j)^{\rho}=\left(i^{\rho}, j^{\rho}\right)$. When we let $h \in H$ act on the vertices 
of $X$ as $(i, j)^{h}=(i, j)^{\rho^{h}}$, then the cyclic group $H$ acts semiregularly on the digraph $X$. Let $U_{0}, \ldots, U_{m-1}$ be the orbits of $H$ on the vertex set $V(X)$. In particular, for $k=1, \ldots, m-2$ let $U_{k}=\operatorname{Orb}_{H}((0, k))$, and let

$$
\begin{aligned}
U_{0} & =\operatorname{Orb}_{H}((m-1,0))=\{(m-1, i) \mid i=0, \ldots, m-2\} \\
U_{m-1} & =\operatorname{Orb}_{H}((0, m-1))=\{(i, m-1) \mid i=0, \ldots, m-2\} .
\end{aligned}
$$

Now define the vertex $u_{i} \in U_{i}$ as

$$
\begin{aligned}
u_{0} & =(m-1,1), \\
u_{i} & =\left(y_{i-1}, y_{i}+1\right), i=1, \ldots, m-2, \\
u_{m-1} & =\left(y_{m-2}, m-1\right),
\end{aligned}
$$

where $y_{i}$ are the elements in $H$ defined in the previous section, and we let $z_{i}=-y_{i}$. Note that the above definition is correct because $y_{i}+1-y_{i-1}=i$ holds in $H$ for all $i=1, \ldots, m-2$.

Let $\left(S_{i, j}\right)$ be the symbol of $X$ relative to $\left(H ; u_{0}, \ldots, u_{m-1}\right)$. We will prove that the element $S_{i, j}$ of the symbol of $X$ coincides with the sets $y_{i}+z_{j-1}+B=\left\{1+y_{i}+z_{j-1}\right\}$ when $i, j \in\{1, \ldots, m-2\}$, that is, with the element in the symbol of $\Gamma$. By definition, $S_{i, j}=\left\{\ell \mid u_{i} u_{j}^{\rho^{\ell}} \in A(X)\right\}$. Also, $u_{j}^{\rho^{\ell}}=\left\{y_{j-1}+\ell, y_{j}+\ell+1\right\}$, showing that $y_{i}+1=y_{j-1}+\ell$, and thus, $S_{i, j}=\left\{y_{i}+z_{j-1}+1\right\}$.

To finish the proof, we need to show that also the sets $S_{i, 0}, S_{i, m-1}, S_{0, j}$ and $S_{m-1, j}$ coincide with the elements of the symbol of $\Gamma$. Observe that for any $i=0, \ldots, m-2$ there are no arcs from the vertices in $U_{i}$ to the vertices in $U_{0}$, and so $S_{i, 0}=\varnothing$ in all these cases. On the other hand, we have $S_{m-1,0}=H=\{0,1, \ldots, m-2\}$, since from any vertex in $U_{m-1}$ there exist arcs to every vertex in $U_{0}$, and since there are no arcs from vertices in $U_{m-1}$ to vertices in $U_{j}$, where $j=1, \ldots, m-1$, we have $S_{m-1, j}=\emptyset$ for every $j=1, \ldots, m-1$.

We are left with the sets $S_{i, m-1}, i=0, \ldots, m-2$ and $S_{0, j}, j=1, \ldots, m-1$. An argument similar to the one used in the general case works nicely here as well. We need to prove that $S_{i, m-1}$ coincides with $y_{i}+z_{m-2}+B=\left\{1+y_{i}+z_{m-2}\right\}$. This follows because $S_{i, m-1}$ contains $\ell$ exactly when there is an arc from $u_{i}$ to $u_{m-1}^{\rho^{\ell}}=$ $\left(y_{m-2}+\ell, m-1\right)$. As $u_{i}$ has second coordinate $y_{i}+1$, it follows that $\ell=1+y_{i}+z_{m-2}$. Finally, we need to prove that $S_{0, j}$ coincides with $z_{j-1}+B=\left\{1+z_{j-1}\right\}$. Now, $S_{0, j}$ contains $\ell$ exactly when there is an arc from $u_{0}=(m-1,1)$ to $u_{j}^{\rho^{\ell}}$, and as the latter vertex has first coordinate $y_{j-1}+\ell$, it follows that $\ell=1+z_{j-1}$, which completes the proof.

We set further notation for the DSRG graph $\Gamma$ arising from our PSF $\left(S_{i, j}\right)$. The cyclic group $H$ acts on the vertices of $\Gamma$ as an $(m, n)$-semiregular group of automorphisms where $n=|H|$. Let $U_{i}$ be the orbits of $H$, and $u_{i}$ be a fixed vertex in $U_{i}$ for all $i=0, \ldots, m-1$. We write the vertices in $U_{i}$ as $u_{i, h}=u_{i}^{h}$ where $h$ runs over the group $H$. (Note that the element $u_{i, h}$ was denoted by $h_{i}$ in Remark 2.3. We use this notation now to facilitate readability in the next proofs.) Consequently, we have 
$u_{i, h}^{k}=u_{i, h+k}$ for all $i=0, \ldots, m-1$ and $h, k \in H$. The digraph $\Gamma$ has symbol $\left(S_{i, j}\right)$ relative to $\left(H ; u_{0}, \ldots, u_{m-1}\right)$. Note that this yields

$$
\Gamma\left(u_{i, h}\right)=\left\{u_{j, x} \mid j=0, \ldots, m-1, x \in S_{i, j}+h\right\},
$$

where $\Gamma\left(u_{i, h}\right)$ denotes the set of out-neighbours of $u_{i, h}$. For a subgroup $G \leq \operatorname{Aut}(\Gamma)$ and a vertex $v \in V(\Gamma)$, we denote by $G_{v}$ the stabilizer of $v$ in $G$. In the following two theorems, $D_{2 \alpha-1}$ denotes the dihedral group with cardinality $4 \alpha-2$, and $C_{\alpha m-1}$ denotes the cyclic group with cardinality $\alpha m-1$.

Theorem 4.2 Let $\Gamma=\Gamma\left(\mathbb{Z}_{2 \alpha-1}, \alpha\right.$, 2). If $\alpha>1$, then $\operatorname{Aut}(\Gamma) \cong D_{2 \alpha-1}$.

Proof In this case, $n=2 \alpha-1$ and $S=S_{0,0}=\{1,2, \ldots, \alpha-1\} \subseteq H=\mathbb{Z}_{n}$, $S_{1,1}=-S, T=S_{0,1}=\{1, \ldots, \alpha\}=S \cup\{\alpha\}$ and $S_{1,0}=-T$. For the sake of simplicity, we write $V=U_{0}, W=U_{1}$, and also $v_{i}=u_{0, i}$ and $w_{i}=u_{1, i}$. We claim that $G=\operatorname{Aut}(\Gamma) \cong D_{n}$. The group $G$ preserves undirected edges. But the graph induced by the undirected edges is a connected bipartite graph with bipartition $\{V, W\}$, and we deduce that $\{V, W\}$ is a block system for $G$. Now we can see that the group $G$ contains a subgroup isomorphic to the dihedral group $D_{n}$. To prove it, we can check that the permutation $\tau$ given by the rules:

$$
v_{i}^{\tau}=w_{-i}, \quad w_{i}^{\tau}=u_{-i}
$$

is an automorphism of $\Gamma$ and that $\tau$ and $H$ generate a dihedral group with cardinality $2 n$. Now, to finish the proof of the theorem it suffices to prove that for each $v \in V(\Gamma)$ the stabilizer $G_{v}$ is trivial. Since $\Gamma$ is vertex-transitive, it suffices to show this for $v=v_{0}$. The subgraph induced by $V$ is a tournament. There are two kinds of triangles: directed triangles, where each vertex has one incoming and one outgoing arc, and transitive triangles (one vertex has two incoming arcs, one vertex has two outgoing arc, and one vertex has one outgoing arc and one incoming arc).

Let us count the number of directed triangles containing particular vertices (arcs). It can be seen that the arc $v_{0} v_{1}$ is on just one directed triangle $v_{0} v_{1} v_{\alpha}$. Similarly, the arc $v_{n-1} v_{0}$ is on just one directed triangle, namely the triangle $v_{n-1} v_{0} v_{\alpha-1}$. Next, the arc $v_{0} v_{2}$ is on two directed triangles $v_{0} v_{2} v_{\alpha}$ and $v_{0} v_{2} v_{\alpha+1}$. (Similarly, the arc $v_{n-2} v_{0}$ is on two directed triangles.) Continuing this way, we can see that the $\operatorname{arcs} v_{0} v_{i}\left(v_{n-i} v_{0}\right)$, $i \in\{1, \ldots, \alpha-1\}$, are contained in $i$ directed triangles. Consequently, $G_{v}$ fixes the sets $\left\{v_{i}, v_{n-i}\right\}$, for $i \in\{1, \ldots, \alpha-1\}$. But an automorphism interchanging some $v_{i}$ and $v_{n-i}$ would reverse the orientation of the $\operatorname{arcs} v_{n-i} v_{0}$ and $v_{0} v_{i}$. Hence, we must have $G_{v}=1$.

Theorem 4.3 Let $\Gamma=\Gamma\left(\mathbb{Z}_{\alpha m-1}, \alpha, m\right)$. If $\alpha>1$ and $m>2$, then $\operatorname{Aut}(\Gamma) \cong C_{\alpha m-1}$.

We will prove the theorem in the end of the section following five preparatory lemmas. For the rest of the section, we set $a=m-1$. Note that $n=\alpha a+\alpha-1$.

Lemma 4.4 With the notation as above, let $\alpha>1, a>1$ and $p \in H$. Then

(i) $B=B+p$ if and only if $p=0$. 
(ii) $A=A+p$ if and only if $p=0$.

(iii) if also $\alpha>2$, then $|A \cap A+p|=|A|-1$ if and only if $p=a$ or $p=n-a$.

Proof (i) Suppose $B=B+p$, and let $b \in B$. Then $b+u p \in B$ for all $u \in \mathbb{Z}^{+}$, and hence $B$ is a union of $P$-cosets, where $P=\langle p\rangle$. Then $|P|$ divides both $|B|=\alpha$ and $|H|=\alpha a+\alpha-1$, hence $|P|=1$ and $p=0$.

(ii) The set $A$ is given as $A=\{a, \ldots,(\alpha-1) a\}$. Since $n=\alpha a+\alpha-1, \alpha a \neq 0$ in $H$, and it follows that $\alpha a$ is not in $A$. Suppose that $A=A+p$. Then $a+p \in A$, and $s a=a+p$ for some $s \in\{1, \ldots, \alpha-1\}$, and hence, $\alpha a=s^{\prime} a+p$ with $s^{\prime} \in\{2, \ldots, \alpha\}$. If $p \neq 0$, then $s^{\prime} \neq \alpha$ and $\alpha a \in A+p=A$, a contradiction. Thus, $p=0$, and (ii) follows.

(iii) Suppose that $p \notin\{0, a, n-a\}$. We have to show that in this case $|A \cap A+p|<$ $|A|-1$. Let $a+p \in A$. Since $p \neq 0$ and $p \neq a$, we get both $\alpha a$ and $(\alpha+1) a$ are in $A+p$. As these elements are not in $A$, we are done. Let $a+p \notin A$. If $2 a+p \in A$, then since $p \neq n-a$, we find $(2 a+p)-a \in A$, contradicting that $p+a \notin A$. Thus, $a+p, 2 a+p \notin A$, and so $|A \cap A+p|<|A|-1$. This completes the proof of the lemma.

Lemma 4.5 With the notation as above, let $\alpha>1, a>1$ and let $v \in \Gamma\left(u_{0}\right)$. Then

$$
\left|\Gamma\left(u_{0}\right) \cap \Gamma(v)\right| \leq n-a-1,
$$

and equality holds if and only if $v=u_{0, a}$ or $v=u_{\alpha-1,(\alpha-1) a+1+z_{\alpha-2}}$ (the latter case occurs only if $\alpha \leq a$ ).

Proof Let $s=(\alpha-1) a+1+z_{\alpha-2}$. Observe that $s \in B+y_{0}+z_{\alpha-2}$, and hence $u_{\alpha-1, s} \in \Gamma\left(u_{0}\right)$. Also, we let $f(v)=\left|\Gamma\left(u_{0}\right) \cap \Gamma(v)\right|$. We compute next $f\left(u_{0, a}\right)$ and $f\left(u_{\alpha-1, s}\right)$. Using (11), we find the expressions

$$
\begin{aligned}
f\left(u_{0, a}\right) & =|A \cap A+a|+\sum_{j=0}^{a-1}\left|B+z_{j} \cap B+z_{j}+a\right|, \\
f\left(u_{\alpha-1, s}\right) & =\left|A \cap A+y_{\alpha-1}+s\right|+\sum_{j=0}^{a-1}\left|B+z_{j} \cap B+y_{\alpha-1}+z_{j}+s\right| \\
& =|A \cap A-a|+\sum_{j=0}^{a-1}\left|B+z_{j} \cap B+z_{j}-a\right| .
\end{aligned}
$$

We find $|A \cap A \pm a|=|A|-1=\alpha-2$. This can be verified easily for $\alpha=2$, and it follows by Lemma 4.4(iii) for $\alpha>2$. Next, $\left|B+z_{j} \cap B+z_{j}+a\right|=\mid(A \cup$ $\{0\}) \cap(A \cup\{\alpha a\}) \mid=\alpha-1$ because $\alpha a \neq 0$ in $H$. Also, $\left|B+z_{j} \cap B+z_{j}-a\right|=$ $|(A \cup\{0\}) \cap(A \backslash\{(\alpha-1) a\} \cup\{0, n-a\})|=\alpha-1$, again because $\alpha a \neq 0$ in $H$. We conclude that $f\left(u_{0, a}\right)=f\left(u_{\alpha-1, s}\right)=\alpha-2+a(\alpha-1)=n-a-1$.

Now, suppose that $v \in \Gamma\left(u_{0}\right)$ such that $v \neq u_{0, a}$ and $v \neq u_{\alpha-1, s}$. We are going to show that $f(v)<n-a-1$.

Case $1 v \in U_{0}$. In this case $\alpha>2$ and $v=u_{0, r a}$ for some $2 \leq r \leq \alpha-1$. Then $f(v)$ satisfies 


$$
f(v)=|A \cap A+r a|+\sum_{j=0}^{a-1}\left|B+z_{j} \cap B+z_{j}+r a\right| .
$$

By Lemma 4.4, $|A \cap A+r a|<\alpha-2$. Now, suppose that $\left|B+z_{j} \cap B+z_{j}+r a\right|=\alpha$. Thus by Lemma 4.4(i), $r a=0$ in $H$, a contradiction. We conclude that $\mid B+z_{j} \cap$ $B+z_{j}+r a \mid \leq \alpha-1$ for all $j$, and hence, $f(v)<n-a-1$.

Case $2 v \in U_{i}, 1 \leq i \leq a-1$. Then $v=u_{i, r a+1+z_{i-1}}$ for some $0 \leq r \leq \alpha-1$, and by (11),

$$
f(v)=\left|A \cap A+y_{i}+r a+1+z_{i-1}\right|+\sum_{j=0}^{a-1}\left|B+z_{j} \cap B+y_{i}+z_{j}+r a+1+z_{i-1}\right| .
$$

Let $t=y_{i}+r a+1+z_{i-1}$. Then $t=r a+i<\alpha a<n$, and thus, $t \neq 0$ in $H$.

Suppose that $\alpha>2$. Then by Lemma 4.4(i), $\left|B+z_{j} \cap B+z_{j}+t\right|<\alpha$. Thus, we are done with this case if we prove that $|A \cap A+t|<\alpha-2$. By Lemma 4.4(iii), this is equivalent to show that $t \neq a$ and $t \neq n-a$. It is easy to see that $t \neq a$. Now, $t=n-a$ if and only if $r a+i=n-a=(\alpha-1) a+\alpha-1$. Notice that the positive integer $t$ is written uniquely as the sum $t=t_{1} a+t_{2}$ with $t_{1} \geq 0$ and $t_{2} \in\{0, \ldots, a-1\}$. Since $\alpha \leq a$, this yields $r=i=\alpha-1$, and so $v=u_{\alpha-1,(\alpha-1) a+1+z_{\alpha-2}}=u_{\alpha-1, s}$, a contradiction.

Now, suppose that $\alpha=2$. Since $t \neq 0$ in $H,|A \cap A+t|=0$. Also, $\mid B+z_{j} \cap B+$ $z_{j}+t|=|\{0, a\} \cap\{t, a+t\} \mid$, where $t=r a+i, r \in\{0,1\}$ and $i \in\{1, \ldots, a-1\}$. It follows that $t \neq a$. Also, since $v \neq u_{\alpha-1, s}$, we find that $i \neq 1$ or $r \neq 1$, and so $t=r a+i \neq a+1=n-a$. We conclude that $\left|B+z_{j} \cap B+z_{j}+t\right|=0$, and so $f(v)=0$. This completes the proof of Case 2 .

Case $3 v \in U_{a}$. Then $v=u_{a, r a+1+z_{a-1}}$ for some $0 \leq r \leq \alpha-1$, and by (11),

$$
f(v)=\left|A \cap C+r a+1+z_{a-1}\right|+\sum_{j=0}^{a-1}\left|B+z_{j} \cap D+z_{j}+r a+1+z_{a-1}\right| .
$$

Then $\left|B+z_{j} \cap D+z_{j}+r a+1+z_{a-1}\right|=|A \cup\{0\} \cap\{0, \ldots, \alpha-2\}+(r+\alpha) a|$. Suppose that $\alpha>2$ and that the latter cardinality is equal to $\alpha-1$. Since $\alpha>2$, we find that $A \cup\{0\}$ contains two elements whose difference is 1 in $H$. Thus, there exists $1 \leq p \leq \alpha-1$ such that $p a= \pm 1$ in $H$. Therefore, as positive integers, $p a \equiv \pm 1(\bmod n)$, and since $p a<n$, we get $p a=1$ or $p a=n-1$. On the other hand, $1<a \leq p a<\alpha a=n-\alpha+1 \leq n-2$, a contradiction. We conclude that $\left|B+z_{j} \cap D+z_{j}+r a+1+z_{a-1}\right| \leq \alpha-2$ for all $j$, and from this it follows that $f(v)<n-a-1$.

Now, suppose that $\alpha=2$. In this case, $\left|B+z_{j} \cap D+z_{j}+r a+1+z_{a-1}\right|=$ $|\{0, a\} \cap\{(r+2) a\}|$ with $r=0$ or $r=1$. Since $n=2 a+1$, it follows that $|\{0, a\} \cap\{(r+2) a\}|=0$, and $f(v) \leq\left|A \cap C+r a+1+z_{a-1}\right| \leq 1<a=n-a-1$. This completes the proof of Case 3 . 
Lemma 4.6 With the notation as above, every automorphism in $G_{u_{0}}$ fixes the vertex $u_{0, p a}$ for all $p \geq 0$.

Proof Let $\gamma \in G_{u_{0}}$. By Lemma 4.5, $\gamma$ maps $u_{0, a}$ to itself or to $u_{\alpha-1, s}$, where $s=(\alpha-$ $1) a+1+z_{\alpha-2}$. A straightforward check shows that $u_{0}$ is an out-neighbour of $u_{\alpha-1, s}$, while it is not an out-neighbour of $u_{0, a}$. This with the previous observation gives that $\gamma$ fixes $u_{0, a}$, and thus $G_{u_{0}} \leq G_{u_{0, a}}$. On the other hand, $G_{u_{0, a}}=G_{u_{0}^{a}}=G_{u_{0}}^{a}$, where the latter group is the conjugate of $G_{u_{0}}$ by $a$. (Here $a$ is regarded as an automorphism of $\Gamma$.) Thus for any $p \geq 0, G_{u_{0}}^{p a}=G_{u_{0}}$, which implies $(p a) \gamma(-p a) \in G_{u_{0}}$, hence $\left(u_{0, p a}\right)^{\gamma}=u_{0, p a}$.

In the proof of Theorem 4.3, we will also use some spectral properties of $\Gamma$. Let $\mathbb{C}^{V(\Gamma)}$ be the vector space of complex row vectors of length $|V(\Gamma)|=m n$, whose coordinates are labelled by the vertices in $V(\Gamma)$. Given a vector $\mathbf{v}=\left(v_{1}, \ldots, v_{m}\right) \in$ $\mathbb{C}^{m}$ and a character $\chi \in \operatorname{Irr}(H)$, the tensor product $\mathbf{v} \otimes \chi$ is the vector in $\mathbb{C}^{V(\Gamma)}$ whose entry, labelled by $u_{i, h}$, is equal to $v_{i} \chi(h)$. Here and in what follows $\operatorname{Irr}(H)$ denotes the set of all irreducible characters of $H$, and $\chi_{0}$ will denote the trivial character.

Let $M$ be an adjacency matrix of $\Gamma$ whose rows and columns are labelled with $V(\Gamma)$ in accordance with the labelling of the vectors in $\mathbb{C}^{V(\Gamma)}$. It was shown in Proposition 3.1 in [8] that the spectrum of $\Gamma$ is equal to the union of all spectra of the matrices $\overline{A_{\chi}}$ (counting multiplicities), where $A_{\chi}=\left(\chi\left(S_{i, j}\right)\right), \chi \in \operatorname{Irr}(H)$ and $\overline{A_{\chi}}$ is the matrix obtained from $A_{\chi}$ after taking the complex conjugate of all entries. Furthermore, it was shown in Proposition 3.2 in [8] that, if $\theta$ is an eigenvalue of $\overline{A_{\chi}}$ with eigenvector $\mathbf{v}$, then $\mathbf{v} \otimes \chi$ is an eigenvector of $M$ corresponding to $\theta$ (regarded as an eigenvalue of $\Gamma$ ).

Lemma 4.7 With the notation as above, let $\alpha>1$. Then $\Gamma$ has three eigenvalues: $n,-1$ and 0 , and the corresponding multiplicities are $1, n$ and $m n-n-1$, respectively. Furthermore, the eigenspace corresponding to the eigenvalue -1 is spanned by the following vectors: $(a,-1, \ldots,-1) \otimes \chi_{0}$, and $\left(\chi(-A), \chi\left(-B-z_{0}\right), \ldots, \chi(-B-\right.$ $\left.\left.z_{a-1}\right)\right) \otimes \chi, \chi \in \operatorname{Irr}(H) \backslash\left\{\chi_{0}\right\}$.

Proof We define the following vectors in $\mathbb{C}^{m}$ :

$$
\begin{aligned}
\mathbf{v}_{1} & =(1, \ldots, 1), \\
\mathbf{v}_{2} & =(a,-1, \ldots,-1), \\
\mathbf{v}_{\chi} & =\left(\chi(A), \chi\left(B+z_{0}\right), \ldots, \chi\left(B+z_{a-1}\right)\right), \chi \in \operatorname{Irr}(H) \backslash\left\{\chi_{0}\right\} .
\end{aligned}
$$

The spectrum of $\Gamma$ is equal to the union of all spectra of $\overline{A_{\chi}}, \chi \in \operatorname{Irr}(H)$, where

$$
A_{\chi}=\left(\begin{array}{cccc}
\chi(A) & \chi\left(B+z_{0}\right) & \ldots & \chi\left(B+z_{a-1}\right) \\
\chi\left(y_{1}\right) \chi(A) & \chi\left(y_{1}\right) \chi\left(B+z_{0}\right) & \ldots & \chi\left(y_{1}\right) \chi\left(B+z_{a-1}\right) \\
\vdots & \vdots & \vdots & \vdots \\
\chi\left(y_{a-1}\right) \chi(A) & \chi\left(y_{a-1}\right) \chi\left(B+z_{0}\right) & \ldots & \chi\left(y_{a-1}\right) \chi\left(B+z_{a-1}\right) \\
\chi(C) & \chi\left(D+z_{0}\right) & \ldots & \chi\left(D+z_{a-1}\right)
\end{array}\right) .
$$


Let $\chi=\chi_{0}$ be the trivial character. Simple computations give $\mathbf{v}_{1} A_{\chi_{0}}=n \mathbf{v}_{1}$ and $\mathbf{v}_{2} A_{\chi}=-\mathbf{v}_{2}$. The matrix $A_{\chi}$ has rank 2 , and we conclude that $\overline{A_{\chi_{0}}}=A_{\chi_{0}}$ has eigenvalues $n,-1$ and 0 , and the corresponding multiplicities are 1,1 and $m-2$.

Now, suppose that $\chi \neq \chi_{0}$. We have shown in the proof of Proposition 3.2 that

$$
(A+H) \ominus\left(z_{a-1}+D+A\right) \oplus\left(z_{a-1}+B+C\right)=\alpha H
$$

holds in the group ring $\mathbb{Z}[H]$. Apply $\chi$ to both sides of this equation, this yields $\chi(D) \chi(A)=\chi(B) \chi(C)$. Notice that $\chi(B) \neq 0$. Indeed, $\chi(B)=\chi(1) \sum_{i=0}^{\alpha-1} \xi^{i}$, where $\xi=\chi(a)$ is an $n$th complex root of unity. Clearly, $\chi(B) \neq 0$ if $\xi=1$. Suppose that $\xi \neq 1$. Then $\chi(B)=0$ if and only if $\xi^{\alpha}=1$. On the other hand, $\operatorname{gcd}(n, \alpha)=1$ (recall that $n=\alpha a+\alpha-1$ ), and this shows that $\xi^{\alpha} \neq 1$, and hence, $\chi(B) \neq 0$. It follows that the first row of $A_{\chi}$ is $\mathbf{v}_{\chi}, \mathbf{v}_{\chi}$ is a nonzero vector, and each other row is a multiple of $\mathbf{v}_{\chi}$. Thus, $A_{\chi}$ has rank 1 and 0 is an eigenvalue of $A_{\chi}$ with multiplicity $m-1$. Furthermore, we compute

$$
\begin{aligned}
\mathbf{v}_{\chi} A_{\chi} & =\left(\chi(A)+\sum_{j=1}^{a-1} \chi\left(B+y_{j}+z_{j-1}\right)+\chi\left(D+z_{a-1}\right)\right) \mathbf{v}_{\chi} \\
& =\chi\left(A \oplus\{0,1, \ldots, a-2\}+B \oplus z_{a-1}+D\right) \mathbf{v}_{\chi}=\chi(H \ominus\{0\}) \mathbf{v}_{\chi}=-\mathbf{v}_{\chi} .
\end{aligned}
$$

The equality preceding the last one follows from (9). To sum up, $A_{\chi}$ has eigenvalues -1 and 0 , and the corresponding multiplicities are 1 and $m-1$. Thus, $\overline{A_{\chi}}$ has the same spectrum, and the first statement of the lemma follows.

Notice that the vector $\overline{\mathbf{v}_{\chi}}=\left(\chi(-A), \chi\left(-B-z_{0}\right), \ldots, \chi\left(-B-z_{a-1}\right)\right)$ becomes an eigenvector of $\overline{A_{\chi}}$ with corresponding eigenvalue -1 . Also, we have shown above that $\mathbf{v}_{2}$ is an eigenvector of $A_{\chi_{0}}$ with corresponding eigenvalue -1 . In view of these and the paragraph before the lemma, we find that each vector given in the lemma is an eigenvector of $M$ with corresponding eigenvalue -1 . Therefore, we are done if these vectors are also independent. This follows from the well-known fact that the characters in $\operatorname{Irr}(H)$ form a basis of the vector space of all complex-valued functions on $H$, and $\chi(B) \neq 0$ whenever $\chi \neq \chi_{0}$.

Given a subspace $\mathbf{V}$ of $\mathbb{C}^{V(\Gamma)}$, let $\mathbf{V}^{\perp}$ denote its orthogonal complement with respect to the inner product $\langle\mathbf{v}, \mathbf{u}\rangle=\mathbf{v} \overline{\mathbf{u}}^{T}$, that is, $\mathbf{V}^{\perp}=\left\{\mathbf{u} \in \mathbb{C}^{V(\Gamma)}: \mathbf{v} \overline{\mathbf{u}}^{T}=0\right.$ for all $\left.\mathbf{v} \in \mathbf{V}\right\}$. For $\gamma \in \operatorname{Aut}(\Gamma)$, the permutation matrix $P_{\gamma}$ is the $m n \times m n$ matrix whose rows and columns are labelled by the vertices of $\Gamma$ in accordance with the labelling of the vectors in $\mathbb{C}^{V(\Gamma)}$, and for $u$ and $v \in V(\Gamma)$,

$$
\left(P_{\gamma}\right)_{u, v}= \begin{cases}1 & \text { if } u^{\gamma}=v \\ 0 & \text { otherwise }\end{cases}
$$

Let $\mathbf{v} \in \mathbb{C}^{V(\Gamma)}$ and $\mathbf{v}^{\prime}=\mathbf{v} P_{\gamma}$. If $\mathbf{v}_{u}$ and $\mathbf{v}_{u}^{\prime}$ denote the entry of $\mathbf{v}$ and $\mathbf{v}^{\prime}$, respectively, labelled by the vertex $u \in V(\Gamma)$, then it holds $\mathbf{v}_{u}^{\prime}=\mathbf{v}_{u^{\gamma^{-1}}}$. It is well known that each eigenspace $\mathbf{V}$ of $M$ is $\operatorname{Aut}(\Gamma)$-invariant, that is, for every $\mathbf{v} \in \mathbf{V}$ and $\gamma \in \operatorname{Aut}(\Gamma)$, 
$\mathbf{v} P_{\gamma} \in \mathbf{V}$. Note that the orthogonal complement $\mathbf{V}^{\perp}$ is also $\operatorname{Aut}(\Gamma)$-invariant. Indeed, if $\mathbf{v} \in \mathbf{V}$ and $\mathbf{u} \in \mathbf{V}^{\perp}$, then $\mathbf{v}\left(\overline{\mathbf{u} P_{\gamma}}\right)^{T}=\mathbf{v}{\overline{P_{\gamma}}}^{T} \overline{\mathbf{u}}^{T}=\mathbf{v}\left(P_{\gamma}\right)^{-1} \overline{\mathbf{u}}^{T}=\left(\mathbf{v} P_{\gamma^{-1}}\right) \overline{\mathbf{u}}^{T}=0$.

Lemma 4.8 With the notation as above, let $\alpha>1$ and $\boldsymbol{V}$ be the eigenspace of $M$ corresponding to the eigenvalue 0 . Then $\boldsymbol{V}^{\perp}$ is spanned by the following vectors: $(0, \ldots, 0,1) \otimes \chi_{0},(1, \ldots, 1) \otimes \chi_{0}$, and $v(\chi) \otimes \chi$, where $\chi \in \operatorname{Irr}(H) \backslash\left\{\chi_{0}\right\}$ and $v(\chi)=\left(v(\chi)_{1}, v(\chi)_{2}, \ldots, v(\chi)_{m}\right)$ satisfies $v(\chi)_{1}=1$.

Proof For $\chi \in \operatorname{Irr}(H)$, let $\mathbf{V}(\chi)$ denote the eigenspace of $\overline{A_{\chi}}$ corresponding to the eigenvalue 0 . We find next a basis of $\mathbf{V}(\chi)$ for all $\chi$.

It is not hard to find that $\mathbf{V}\left(\chi_{0}\right)$ is spanned by the $m-2$ vectors

$$
(1,-1,0, \ldots, 0), \ldots,(1,0, \ldots,-1,0)
$$

Now, suppose that $\chi \in \operatorname{Irr}(H)$ such that $\chi \neq \chi_{0}$. Define

$$
\mathbf{u}= \begin{cases}(0, \ldots, 0,1) & \text { if } \chi(D)=0 \\ (1,0, \ldots, 0,-\overline{\chi(B)} / \overline{\chi(D)}) & \text { if } \chi(D) \neq 0 .\end{cases}
$$

It is not hard to verify that $\mathbf{V}(\chi)$ is spanned by $\mathbf{u}$ and the $m-2$ vectors

$$
\left(1,-\chi\left(y_{1}\right), 0, \ldots, 0\right), \ldots,\left(1,0, \ldots,-\chi\left(y_{a-1}\right), 0\right) .
$$

Then, for $\chi \in \operatorname{Irr}(H) \backslash\left\{\chi_{0}\right\}$, define

$$
v(\chi)= \begin{cases}\left(1, \chi\left(y_{1}\right), \ldots, \chi\left(y_{a-1}\right), 0\right) & \text { if } \chi(D)=0 \\ \left(1, \chi\left(y_{1}\right), \ldots, \chi\left(y_{a-1}\right), \chi(D) / \chi(B)\right) & \text { if } \chi(D) \neq 0\end{cases}
$$

We finish the proof by showing that $\mathbf{V}^{\perp}$ is spanned by $(0, \ldots, 0,1) \otimes \chi_{0},(1, \ldots, 1)$ $\otimes \chi_{0}$ and the $n-1$ vectors $v(\chi) \otimes \chi$. First, it can be shown, using the orthogonality of characters, that each of the latter vectors is orthogonal to the vectors obtained from $\mathbf{u}$ and from any vector in (12) and (13) in the way described in the paragraph before Lemma 4.7. This implies that our vectors belong to $\mathbf{V}^{\perp}$. Second, these are also independent, which can be verified using the independence of characters. Finally, $\operatorname{dim}(\mathbf{V})=m n-n-1$, and hence $\operatorname{dim}\left(\mathbf{V}^{\perp}\right)=m n-\operatorname{dim}(\mathbf{V})=n+1$; all these yield the lemma.

Everything is prepared to settle Theorem 4.3.

Proof of Theorem 4.3 Let $\gamma \in G=\operatorname{Aut}(\Gamma), P_{\gamma}$ be the permutation matrix defined by $\gamma$, and let $U_{i}^{\gamma}$ denote the image of the $H$-orbit $U_{i}$ under $\gamma$ for $i \in\{0, \ldots, a\}$. Clearly, $H \leq G$. We derive the equality $G=H$ through a series of five claims.

Claim $1 U_{0}^{\gamma}=U_{0}$ whenever $\gamma$ fixes $u_{0}$. 
Let $\mathbf{V}$ be the eigenspace of $M$ corresponding to the eigenvalue -1 . By Lemma 4.7, the vector $\mathbf{v}=(a,-1, \ldots,-1) \otimes \chi_{0}$ is in $\mathbf{V}$, and thus $\mathbf{v} P_{\gamma} \in \mathbf{V}$, and so

$$
\mathbf{v} P_{\gamma}=c_{\chi_{0}} \mathbf{v}+\sum_{\chi \in \operatorname{Irr}(H) \backslash\left\{\chi_{0}\right\}} c_{\chi}\left(\left(\chi(-A), \chi\left(-B-z_{0}\right), \ldots, \chi\left(-B-z_{a-1}\right)\right) \otimes \chi\right)
$$

holds with unique constants $c_{\chi}$. For a subset $X \subseteq H$, let $\mathbf{1}_{X}$ denote the characteristic vector of $X$. Divide $\mathbf{v} P_{\gamma}$ into $m$ blocks, each of length $n$, in the way described in the paragraph after Lemma 4.6 and regarded as a complex-valued function on $H$. We can write $\mathbf{v} P_{\gamma}=\left(m \mathbf{1}_{T_{0}}-\mathbf{1}_{H}, \ldots, m \mathbf{1}_{T_{a}}-\mathbf{1}_{H}\right)$ with suitable subsets $T_{i} \subseteq H$. Notice that Claim 1 is equivalent to saying that $T_{0}=H$ and $T_{i}=\emptyset$ whenever $i>0$.

Now, (14) splits into $m$ linear combinations

$$
\begin{aligned}
& m \mathbf{1}_{T_{0}}-\mathbf{1}_{H}=\left(a c_{\chi 0}\right) \chi_{0}+\sum_{\chi \in \operatorname{Irr}(H) \backslash\left\{\chi_{0}\right\}}\left(c_{\chi} \chi(-A)\right) \chi \\
& m \mathbf{1}_{T_{i}}-\mathbf{1}_{H}=-c_{\chi_{0}} \chi_{0}+\sum_{\chi \in \operatorname{Irr}(H) \backslash\left\{\chi_{0}\right\}}\left(c_{\chi} \chi\left(-B-z_{i-1}\right)\right) \chi, i=1, \ldots, a .
\end{aligned}
$$

It is well known that in the vector space of all complex-valued functions on $H$ the characters in $\operatorname{Irr}(H)$ form an orthonormal basis with respect to the inner product $[f, g]=\frac{1}{n} \sum_{h \in H} f(h) \overline{g(h)}$. Now, by (15) and (16), respectively,

$$
\begin{aligned}
& \frac{m}{n}\left|T_{0}\right|-1=\left[\chi_{0}, m \mathbf{1}_{T_{0}}-\mathbf{1}_{H}\right]=a c_{\chi_{0}} \\
& \frac{m}{n}\left|T_{i}\right|-1=\left[\chi_{0}, m \mathbf{1}_{T_{i}}-\mathbf{1}_{H}\right]=-c_{\chi_{0}} .
\end{aligned}
$$

These show that $\left|T_{i}\right|=\left|T_{1}\right|$ for all $i>0$. Let $t_{0}=\left|T_{0}\right|$ and $t_{1}=\left|T_{1}\right|$. It is easily seen that $t_{0}+a t_{1}=n$. Let $\chi \in \operatorname{Irr}(H)$ such that $\chi \neq \chi_{0}$. Then by (15) and (16) with $i=1$, respectively,

$$
\begin{aligned}
& \frac{m}{n} \chi\left(-T_{0}\right)=\left[\chi, m \mathbf{1}_{T_{0}}-\mathbf{1}_{H}\right]=c_{\chi} \chi(-A) \\
& \frac{m}{n} \chi\left(-T_{1}\right)=\left[\chi, m \mathbf{1}_{T_{1}}-\mathbf{1}_{H}\right]=c_{\chi} \chi(-B) .
\end{aligned}
$$

Recall that $\chi\left(T_{i}\right)$ is defined to be 0 when $T_{i}=\emptyset$. Notice that, up to now, we did not use the assumption that $\gamma$ fixes $u_{0}$, and thus, all above equalities hold when replacing $\gamma$ with any automorphism in $G$. These will be used later in the proof of Claim 2 . Let $\langle a\rangle$ be the subgroup of $H$ generated by the element $a$, let $x \in\langle a\rangle$ and $v=u_{0, x}$. Since $u_{0}$ is fixed by $\gamma, v^{\gamma}=v$, see Lemma 4.6. Therefore, $\left(\mathbf{v} P_{\gamma}\right)_{v}=\mathbf{v}_{v^{-1}}=\mathbf{v}_{v}=a$. This gives $\langle a\rangle \subseteq T_{0}$. Assume to the contrary that $t_{1}=\left|T_{1}\right|>0$.

Now, as both $T_{0}$ and $T_{1}$ are non-empty sets, it follows from (17) and (18) that $\chi\left(T_{0}+B\right)=\chi\left(T_{1}+A\right)$ holds in the group ring $\mathbb{Z}[H]$ for every non-trivial character $\chi$. This implies that

$$
T_{0}+B=\left(T_{1}+A\right) \oplus q H
$$


for some integer $q$. Apply the trivial character $\chi_{0}$ to both sides, this gives $\alpha t_{0}=$ $(\alpha-1) t_{1}+q n$. Now, using also that $n=\alpha a+\alpha-1=t_{0}+a t_{1}$, we deduce that $q=\alpha-t_{1}$.

As a $\mathbb{Z}[H]$-element, $T_{0}+B$ is expressed as the formal sum $T_{0}+B=\sum_{x \in H} a_{x}^{\star} x$ with suitable nonnegative integers $a_{x}^{\star}$. Then we find $a_{\alpha a+1}^{\star}=\left|T_{0} \cap-B+\alpha a+1\right|=$ $|B|=\alpha$ because $-B+\alpha a+1 \subseteq\langle a\rangle \subseteq T_{0}$. Let us compute $a_{\alpha a+1}^{\star}$ using the equality $T_{0}+B=\left(T_{1}+A\right) \oplus q H$. From this, we find $a_{\alpha a+1}^{\star}=\left|T_{1} \cap-A+\alpha a+1\right|+q$. Suppose that $T_{1} \cap-A+\alpha a+1$ contains some element $j$, and let $w=u_{1, j}$. As $-A+\alpha a+1 \subset B, j \in B$, and thus $w \in \Gamma\left(u_{0}\right)$. Since, by Lemma 4.6, $\Gamma\left(u_{0}\right) \cap U_{0}$ is fixed by $\gamma$, it follows that $w^{\gamma^{-1}} \notin U_{0}$, and thus, $\left(\mathbf{v} P_{\gamma}\right)_{w}=\mathbf{v}_{w^{\gamma}}=-1$. This gives $j \notin T_{1}$, which, however, contradicts that $j$ was chosen from $T_{1} \cap-A+\alpha a+1$. We conclude that $T_{1} \cap-A+\alpha a+1=\emptyset$, and $a_{\alpha a+1}^{\star}=q$. On the other hand, we have already shown that $a_{\alpha a+1}^{\star}=\alpha$, and so finally get $t_{1}=\alpha-q=0$, a contradiction.

Claim $2 U_{0}^{\gamma}=U_{0}$.

Assume to the contrary that $U_{0}^{\gamma} \neq U_{0}$. We show first that $U_{0}^{\gamma} \cap U_{0}=\emptyset$. Now, suppose that this does not hold, or equivalently, there exist $u_{0, j}$ and $u_{0, j^{\prime}}$ in $U_{0}$ such that $u_{0, j}^{\gamma}=u_{0, j^{\prime}}$. Let $\gamma^{\prime}=j \gamma\left(-j^{\prime}\right)$, here we regard $j$ and $j^{\prime}$ as automorphisms of $\Gamma$. It follows that $\gamma^{\prime}$ fixes $u_{0}$, and thus by Claim $1, U_{0}^{\gamma^{\prime}}=U_{0}$. On the other hand, $U_{0}^{\gamma^{\prime}}=U_{0}^{\gamma\left(-j^{\prime}\right)}=\left(U_{0}^{\gamma}\right)^{-j^{\prime}}$, hence $U_{0}^{\gamma}=U_{0}^{j^{\prime}}=U_{0}$, a contradiction. Notice that, since $\gamma$ was chosen arbitrarily from $G$,

$$
U_{0}^{\gamma^{\prime}}=U_{0} \text { or } U_{0}^{\gamma^{\prime}} \cap U_{0}=\emptyset
$$

holds for all $\gamma^{\prime} \in G$. (In other words $U_{0}$ is a block of imprimitivity for $G$.) Now, let us repeat the argument for $\gamma$ and $\mathbf{v} P_{\gamma}$ given above, where $\mathbf{v}=(a,-1, \ldots,-1) \otimes \chi_{0}$. Notice that, $t_{0}+a t_{1}=n$. Here we use that $a>1$ to get $t_{1}<n$ and thus that $T_{1} \neq H$, where $\mathbf{v} P_{\gamma}=\left(m \mathbf{1}_{T_{0}}-\mathbf{1}_{H}, \ldots, m \mathbf{1}_{T_{l}}-\mathbf{1}_{H}\right)$. Since $U_{0}^{\gamma} \cap U_{0}=\emptyset$, it follows that $T_{0}=\emptyset$ and $T_{1} \neq \emptyset$. In particular, $\chi\left(T_{0}\right)=0$ for every non-trivial character $\chi$, and thus $c_{\chi} \chi(A)=0$ holds in (17). Using this and (18), we deduce that $\chi\left(T_{1}+A\right)=0$ holds for any $\chi \neq \chi_{0}$. This gives that $T_{1}+A=q H$ for some positive integer $q$. Since $n=t_{0}+a t_{1}=a t_{1}$, it follows that $t_{1}=n / a$.

By definition, $x \in T_{1}$ if and only if $\left(\mathbf{v} P_{\gamma}\right)_{u_{1, x}}=l$. On the other hand, $\left(\mathbf{v} P_{\gamma}\right)_{u_{1, x}}$ is equal to the coordinate of $\mathbf{v}$ labelled by the vertex $\left(u_{1, x}\right)^{\gamma^{-1}}$, which is equal to $a$ exactly when $u_{1, x} \in U_{0}^{\gamma}$. We find that $T_{1}=\left\{x \in H \mid u_{1, x} \in U_{0}^{\gamma}\right\}$. Fix some $a \in T_{1}$ and let $K=T_{1}-a$. Choose arbitrary $x \in T_{1}$, and let us consider $h=x-a$ acting as an automorphism of $\Gamma$. Then $u_{1, x} \in U_{0}^{\gamma h} \cap U_{0}^{\gamma}$, and hence, by (19), $U_{0}^{\gamma h}=U_{0}^{\gamma}$. Therefore, $u_{1, y+h}=u_{1, y}^{h} \in U_{0}^{\gamma}$ for every $y \in T_{1}$, and thus $T_{1}+x-a=T_{1}+h=T_{1}$ follows. Since the latter equality holds for all $x \in T_{1}$, we further get $K+K=K$. By this and that $0 \in K$, it follows that $K$ is a subgroup of $H$, and so $T_{1}$ is equal to the coset $K+a$. The order $|K|=\left|T_{1}\right|=t_{1}=n / a$, showing that $K$ is the unique subgroup of $H$ of order $n / a$, and therefore, $K=\langle a\rangle$. This together with the facts $T_{1}+A=q H$ and $A \subseteq\langle a\rangle$ imply in turn that $T_{1} \cap\langle a\rangle \neq \emptyset, T_{1}=\langle a\rangle$, and finally $T_{1}=H$, which is a contradiction. 
Claim $3 U_{a}^{\gamma}=U_{a}$.

Let $\mathbf{W}$ be the eigenspace of $M$ corresponding to the eigenvalue 0 . Let $\mathbf{w}=$ $(0, \ldots, 0,1) \otimes \chi_{0}$ and $\mathbf{w}^{\prime}=(1, \ldots, 1) \otimes \chi_{0}$. By Lemma 4.8 , both $\mathbf{w}$ and $\mathbf{w}^{\prime}$ are in $\mathbf{W}^{\perp}$, and thus, $\mathbf{w} P_{\gamma} \in \mathbf{W}^{\perp}$, and

$$
\mathbf{w} P_{\gamma}=c \mathbf{w}+c_{\chi_{0}} \mathbf{w}^{\prime}+\sum_{\chi \in \operatorname{Irr}(H) \backslash\left\{\chi_{0}\right\}} c_{\chi}(v(\chi) \otimes \chi)
$$

holds with unique constants $c$ and $c_{\chi}, \chi \in \operatorname{Irr}(H)$, and the vectors $v(\chi) \otimes \chi$ are as described in Lemma 4.8. As before, $\mathbf{w} P_{\gamma}=\left(\mathbf{1}_{R_{0}}, \ldots, \mathbf{1}_{R_{a}}\right)$ holds with suitable subsets $R_{i} \subseteq H$. Since $v(\chi)_{1}=1$ for each non-trivial character $\chi$, we obtain from (20) that $\mathbf{1}_{R_{0}}=\sum_{\chi \in \operatorname{Irr}(H)} c_{\chi} \chi$. On the other hand, by Claim $2, R_{0}=\emptyset$ and so $\mathbf{1}_{R_{0}}$ is the zero vector. Therefore, $c_{\chi}=0$ for each $\chi \in \operatorname{Irr}(H)$, and in (20) we find $\mathbf{w} P_{\gamma}=\mathbf{w}$, and Claim 3 follows.

Claim 4 If $\gamma$ fixes every vertex in $U_{a}$, then $\gamma$ is the identity mapping.

Let $u_{i, j}$ be any vertex with $i \neq a$, and write $u_{i, j}^{\gamma}=u_{i^{\prime}, j^{\prime}}$. We have to show that $i=i^{\prime}$ and $j=j^{\prime}$. Clearly, $u_{i, j}$ and $u_{i^{\prime}, j^{\prime}}$ have the same out-neighbours in $U_{a}$. This is equivalent to the equality $B+y_{i}+z_{a-1}+j=B+y_{i^{\prime}}+z_{a-1}+j^{\prime}$. By Lemma 4.4(i), $y_{i}+j=y_{i^{\prime}}+j^{\prime}$. If $i=0$, then by Claim 2, $i^{\prime}=0$, and we get $j=j^{\prime}$. We have shown that $\gamma$ fixes every vertex in $U_{0}$.

Now, suppose that $i \neq 0$. By the previous paragraph, $u_{i, j}$ and $u_{i^{\prime}, j^{\prime}}$ must have the same in-neighbours in $U_{0}$. Equivalently, $-B-y_{0}-z_{i-1}+j=-B-y_{0}-z_{i^{\prime}-1}+j^{\prime}$, and thus $-z_{i-1}+j=-z_{i^{\prime}-1}+j^{\prime}$. This together with $y_{i}+j=y_{i^{\prime}}+j^{\prime}$ in turn implies that $i-1=y_{i}+z_{i-1}=y_{i^{\prime}}+z_{i^{\prime}-1}=i^{\prime}-1, i=i^{\prime}$, and $j=j^{\prime}$. This completes the proof of Claim 4 .

Claim $5 G=H$.

In view of Claims 3 and $4, G$ admits a faithful action on $U_{l}$. This implies that $G$ is isomorphic to a subgroup of $\operatorname{Aut}\left(\Gamma^{\prime}\right)$, where $\Gamma^{\prime}$ is the subdigraph of $\Gamma$ induced by the set $U_{a}$. We let $F=\operatorname{Aut}\left(\Gamma^{\prime}\right)$, and for sake of simplicity write $v_{i}$ for the vertex $u_{a, i}=u_{a}^{i}$ for $i \in H$. The group $H$ induces a regular subgroup of $F$, and hence, we are done if we show that $F=H$. It follows that $v_{i}$ has out-neighbours:

$$
\Gamma^{\prime}\left(v_{i}\right)=\left\{v_{x} \mid x \in D+z_{a-1}+i\right\} .
$$

If $\alpha=2$, then $D+z_{a-1}=\{-1\}$. Thus, $\Gamma^{\prime}$ is a directed $n$-cycle, and this shows that $F=H$. Now suppose that $\alpha>2$. Let $F_{v_{i}}$ be the stabilizer of the vertex $v_{i}$ in $F$. Let us consider the intersection $\Gamma^{\prime}\left(v_{0}\right) \cap \Gamma^{\prime}\left(v_{i}\right)$. We find $\left|\Gamma^{\prime}\left(v_{0}\right) \cap \Gamma^{\prime}\left(v_{i}\right)\right|=$ $\left|D+z_{a-1} \cap D+z_{a-1}+i\right|$. Using $n=\alpha a+\alpha-1$, we find $D+z_{a-1}=-\{1, \ldots, \alpha-1\}$. It is not hard to show that $\left|\Gamma^{\prime}\left(v_{0}\right) \cap \Gamma^{\prime}\left(v_{i}\right)\right| \leq \alpha-2$ for all $v_{i} \in \Gamma\left(v_{0}\right)$, and equality holds if and only if $i=-1$. Because of this, every automorphism in $F_{v_{0}}$ must fix also $v_{-1}$. This shows that $F_{v_{0}} \leq F_{v_{-1}}$. It can be proved, repeating the argument in the proof 
of Lemma 4.6, that this implies that $F_{v_{0}}=F_{v_{i}}$ for all vertex $v_{i}$, and so $F_{v_{0}}=1$, and we finally get $F=H$. This completes the proof of the theorem.

Acknowledgements We would like to thank the anonymous referees for valuable comments and suggestions.

\section{References}

1. Araluze, A., Kutnar, K., Martínez, L., Marušič, D.: Edge connectivity in difference graphs and some new constructions of partial sum families. European J. Combin. 32, 352-360 (2011)

2. Araluze, A., Kovács, I., Kutnar, K., Martínez, L., Marušič, D.: Partial sum quadruples and bi-Abelian digraphs. J. Combin. Theory Ser. A 119, 1811-1831 (2012)

3. Bose, R.C.: Strongly regular graphs, partial geometries and partially balanced designs. Pacific J. Math. 13, 389-419 (1963)

4. de Resmini, M.J., Jungnickel, D.: Strongly regular semi-Cayley graphs. J. Algebraic Combin. 1, 171195 (1992)

5. Duval, A.: A directed graph version of strongly regular graphs. J. Combin. Theory Ser. A 47, 71-100 (1988)

6. Gyürki, Š.: Infinite families of directed strongly regular graphs using equitable partitions. Discrete Math. 339, 2970-2986 (2016)

7. http://homepages.cwi.nl/ aeb/math/dsrg/dsrg.html

8. Kovács, I., Malnič, A., Marušič, D., Miklavič, Š.: Transitive group actions: (im)primitivity and semiregular subgroups. J. Algebraic Combin. 41, 867-885 (2015)

9. Kutnar, K., Marušič, D., Miklavič, Š., Šparl, P.: Strongly regular tri-Cayley graphs. European J. Combin. 30, 822-832 (2009)

10. Malnič, A., Marušič, D., Šparl, P., Frelih, B.: Symmetry structure of bicirculants. Discrete Math. 307, 409-414 (2007)

11. Martínez, L., Araluze, A.: New tools for the construction of directed strongly regular graphs: difference digraphs and partial sum families. J. Combin. Theory Ser. B 100, 720-728 (2010)

12. Martínez, L.: Strongly regular m-Cayley circulant graphs and digraphs. Ars Math. Contemp. 8(1), 195-213 (2015)

13. Milies, C.P., Sehgal, S.K.: An Introduction to Group Rings. Kluwer, Dordrecht (2002)

14. Ramras, M., Donovan, E.: The automorphism group of a Johnson graph. SIAM J. Discrete Math. 25, 267-270 (2011)

Publisher's Note Springer Nature remains neutral with regard to jurisdictional claims in published maps and institutional affiliations.

\section{Affiliations}

\section{Ricardo Grande ${ }^{1}$ István Kovács ${ }^{2} \cdot$ Klavdija Kutnar $^{2} \cdot$ Aleksander Malnič $^{3}$. Luis Martínez ${ }^{4,5}$. Dragan Marušič 2,3}

$凶 \quad$ Luis Martínez

luis.martinez@ehu.eus

Ricardo Grande

rgi@mit.edu

István Kovács

istvan.kovacs@upr.si 
Klavdija Kutnar

klavdija.kutnar@upr.si

Aleksander Malnič

aleksander.malnic@guest.arnes.si

Dragan Marušič

dragan.marusic@upr.si

1 Department of Mathematics, Massachusetts Institute of Technology, Cambridge, MA, USA

2 FAMNIT, University of Primorska, Glagoljaška 8, 6000 Koper, Slovenia

3 IMFM, University of Ljubljana, Jadranska 19, 1000 Ljubljana, Slovenia

4 Department of Mathematics, University of the Basque Country UPV/EHU, 48080 Bilbao, Spain

5 Basque Center of Applied Mathematics (BCAM), 48009 Bilbao, Spain 